\begin{tabular}{|r|l|}
\hline \multicolumn{2}{|c|}{ Statistica Sinica Preprint No: SS-2020-0395 } \\
\hline Title & $\begin{array}{l}\text { Sharpening the Rosenbaum Sensitivity Bounds to Address } \\
\text { Concerns About Interactions Between Observed and } \\
\text { Unobserved Covariates }\end{array}$ \\
\hline Manuscript ID & SS-2020-0395 \\
\hline URL & http://www.stat.sinica.edu.tw/statistica/ \\
\hline DOI & $10.5705 /$ ss.202020.0395 \\
\hline Complete List of Authors & $\begin{array}{l}\text { Siyu Heng and } \\
\text { Dylan Small }\end{array}$ \\
\hline Corresponding Author & Dylan Small \\
\hline E-mail & dsmall@wharton.upenn.edu \\
\hline Notice: Accepted version subject to English editing. \\
\hline
\end{tabular}


Statistica Sinica

Sharpening the Rosenbaum Bounds

\title{
Sharpening the Rosenbaum Sensitivity Bounds to Address Concerns About Interactions Between Observed and Unobserved Covariates
}

\author{
Siyu Heng and Dylan S. Small \\ University of Pennsylvania
}

Abstract:

In observational studies, it is typically unrealistic to assume that treatments are randomly assigned, even conditional on adjusting for all observed covariates. Therefore, a sensitivity analysis is often needed to examine how hidden biases due to unobserved covariates would affect inferences on treatment effects. In matched observational studies where each treated unit is matched to one or multiple untreated controls for observed covariates, the Rosenbaum bounds sensitivity analysis is one of the most popular sensitivity analysis models. In this paper, we show that in the presence of interactions between observed and unobserved covariates, directly applying the Rosenbaum bounds will almost inevitably exaggerate the report of sensitivity of causal conclusions to hidden bias. We give sharper odds ratio bounds to fix this deficiency. We illustrate our new method through studying the effect of anger/hostility tendency on the risk of having heart problems. 
Sharpening the Rosenbaum Bounds

Key words and phrases: Causal inference; Gene-environment interaction; Interaction terms; Matching; Observational studies; Sensitivity analysis.

\section{Introduction}

In a randomized experiment, units are randomly assigned to the treatment group or control groups, perhaps by flipping a coin. In contrast, in an observational study, treatments are not randomly assigned to units and differences between the outcomes of the treated and control groups can be a biased estimate of the true treatment effect because of baseline differences between the treated and control groups. Baseline differences that can be captured by observed covariates can often be removed by modelbased adjustments or matching. Among these methods, matching has been extensively used as a nonparametric way of adjusting for the observed covariates in observational studies: each treated unit is matched to one or several controls (i.e., untreated units) on baseline observed covariates such that the treated units and controls are similar in measured confounders as they would be under a randomized experiment, and the comparisons are made within these matched sets (Rubin, 1973; Rosenbaum, 2002, 2010, Hansen, 2004; Stuart, 2010; Zubizarreta et al., 2013; Pimentel et al., 2015, Zubizarreta and Keele, 2017). 
Sharpening the Rosenbaum Bounds

However, there is typically the concern that some important baseline covariates are unobserved, so that the treatment assignments may not be random within each matched set. A sensitivity analysis asks how a departure from random assignment of treatment would affect the causal conclusion drawn from a primary analysis that assumes the treatment is randomly assigned conditional on the observed covariates. Among various sensitivity analysis models in matched observational studies, the Rosenbaum bounds sensitivity analysis (Rosenbaum, 1987, 2002) is one of the most popular. The Rosenbaum bounds sensitivity analysis introduces a uniform sensitivity parameter $\Gamma \geq 1$ bounding the ratio of the odds of treatment within each matched set: the more $\Gamma$ departs from 1 , the more the treatment assignment potentially departs from random assignment in each matched set. Then researchers typically look at the "worst-case" p-value, which is defined as the largest $\mathrm{p}$-value given the sensitivity parameter $\Gamma$ over all possible arrangements of unobserved covariates (i.e., unmeasured confounders) (Rosenbaum, 2002). For examples of studies using the Rosenbaum bounds sensitivity analysis, see Normand et al. (2001), Rosenbaum (2002), Rosenbaum (2004), Heller et al. (2009), Silber et al. (2009), Stuart and Hanna (2013), Zubizarreta et al. (2013), Hsu et al. (2015), Zubizarreta et al. (2016), Ertefaie et al. (2018), Fogarty (2019), Karmakar et al. (2019), Zhao (2019), 
Sharpening the Rosenbaum Bounds

and Zhang et al. (2020). Many other sensitivity analysis models also build on the Rosenbaum bounds sensitivity analysis (e.g., Gastwirth et al., 1998; Ichino et al., 2008; Rosenbaum and Silber, 2009; Nattino and Lu, 2018; Fogarty and Hasegawa, 2019).

In this article, we show that in the presence of any interactions between the observed and unobserved covariates in the logit model of the treatment assignment probability, the Rosenbaum bounds will almost inevitably be loose for some of the matched sets. Interactions between observed and unobserved covariates commonly exist in observational studies. One such setting is the extensively-studied "gene-environment interaction" $(\mathrm{G} \times \mathrm{E})$, where two different genotypes respond to environmental variation in different ways (Ottman, 1996; Caspi et al., 2002). In many studies, such genotypes were not identified, measured, or publicly available, and should be considered as unobserved covariates that can interact with some observed environmental covariates (Pérusse and Bouchard, 1999). Directly applying the Rosenbaum bounds sensitivity analysis in such settings can greatly exaggerate the sensitivity of the causal conclusion to hidden bias. To perform a more informative and less conservative sensitivity analysis in matched studies, we give sharper odds ratio bounds when there is concern about a possible interaction between an observed covariate and an unobserved co- 
Sharpening the Rosenbaum Bounds

variate. We apply our new result to study the causal effect of anger/hostility tendency on the risk of having heart disease. The data used for this work is publicly available at https://www.ssc.wisc.edu/wlsresearch/. The code and codebook used for this work are available at https://github. com/siyuheng/Sharpening-the-Rosenbaum-Bounds

\section{A brief review of the Rosenbaum bounds sensitivity analysis}

We briefly review the classical framework for the Rosenbaum bounds sensitivity analysis for a matched observational study in which each treated unit is matched to one or more controls (Rosenbaum, 2002). There are $I$ matched sets $i=1, \ldots, I$, and matched set $i$ contains $n_{i}\left(n_{i} \geq 2\right)$ units, so $N=\sum_{i=1}^{I} n_{i}$ units in total. In each matched set, one unit received treatment and the others received control. Let $Z_{i j}=1$ if unit $j$ in matched set $i$ received treatment, otherwise let $Z_{i j}=0$. Therefore, we have $\sum_{j=1}^{n_{i}} Z_{i j}=1$ for all $i$. Let $\mathbf{x}_{i j}=\left(x_{i j(1)}, \ldots, x_{i j(K)}\right)^{T}$ denote the $K$ observed covariates and $u_{i j}$ an unobserved covariate of unit $j$ in matched set $i$. The sets are matched for the observed covariates but not for the unobserved covariate, therefore $\mathbf{x}_{i j}=\mathbf{x}_{i j^{\prime}}$ for all $i, j$ and $j^{\prime}$, but possibly $u_{i j} \neq u_{i j^{\prime}}$ if $j \neq j^{\prime}$ Rosenbaum, 2002). Denote the common observed covariates for units in matched sets $i$ as $\mathbf{x}_{i}=\left(x_{i(1)}, \ldots, x_{i(K)}\right)^{T}$, where $\mathbf{x}_{i}=\mathbf{x}_{i j}=\mathbf{x}_{i j^{\prime}}$ for all $i, j, j^{\prime}$. Under the poten- 
tial outcome framework, if unit $j$ in matched set $i$ received treatment (i.e., $Z_{i j}=1$ ), we observe the potential outcome $r_{T i j}$; otherwise (i.e., $Z_{i j}=0$ ), we observe the potential outcome $r_{C i j}($ Neyman, 1923; Rubin, 1974). Therefore, the observed outcome for each $i, j$ is $R_{i j}=Z_{i j} r_{T i j}+\left(1-Z_{i j}\right) r_{C i j}$. Denote the collection of observed outcomes as $\mathbf{R}=\left(R_{11}, \ldots, R_{I n_{I}}\right)^{T}$ and the collection of unobserved covariates as $\mathbf{u}=\left(u_{11}, \ldots, u_{I n_{I}}\right)^{T}$. Write $\mathcal{F}=\left\{\left(r_{T i j}, r_{C i j}, \mathbf{x}_{i j}, u_{i j}\right), i=1, \ldots, I, j=1, \ldots, n_{i}\right\}$, and let $\mathcal{Z}$ be the set of all possible values of $\mathbf{Z}=\left(Z_{11}, \ldots, Z_{I n_{I}}\right)^{T}$ where $\mathbf{Z} \in \mathcal{Z}$ if and only if $\sum_{j=1}^{n_{i}} Z_{i j}=1$ for all $i$. Let $|A|$ denote the number of elements of a finite set $A$, and define the indicator function $\mathbf{1}\{A\}=1$ if $A$ is true, and $\mathbf{1}\{A\}=0$ otherwise. Let $a \gg b$ denote that $a$ is much greater than $b$.

Fisher's sharp null hypothesis of no treatment effect asserts that $H_{0}$ : $r_{T i j}=r_{C i j}$, for all $i, j$. In a randomized experiment, we can assume that $\operatorname{pr}(\mathbf{Z}=\mathbf{z} \mid \mathcal{F}, \mathcal{Z})=1 /|\mathcal{Z}|=1 /\left(\prod_{i=1}^{I} n_{i}\right)$ for all $\mathbf{z} \in \mathcal{Z}$. In a stratified randomized experiment with one unit in each matched set being randomly assigned to treatment, the significance level of a test statistic $T$ being greater than or equal to the observed value $t$ can be computed as

$$
\begin{aligned}
\operatorname{pr}(T \geq t \mid \mathcal{F}, \mathcal{Z}) & =\sum_{\mathbf{z} \in \mathcal{Z}} \mathbf{1}\{T(\mathbf{z}, \mathbf{R}) \geq t\} \operatorname{pr}(\mathbf{Z}=\mathbf{z} \mid \mathcal{F}, \mathcal{Z}) \\
& =\frac{|\{\mathbf{z} \in \mathcal{Z}: T(\mathbf{z}, \mathbf{R}) \geq t\}|}{|\mathcal{Z}|}
\end{aligned}
$$


Sharpening the Rosenbaum Bounds

In an observational study, it is often unrealistic to assume that treatment was randomly assigned, even within a matched set of units with the same observed covariates, due to the possible presence of an unobserved covariate. A sensitivity analysis is therefore needed to determine how departures from random assignment of treatment would affect the causal conclusions drawn from a primary analysis that assumes the treatment is randomly assigned within each matched set. Let $\pi_{i j}=P\left(Z_{i j}=1 \mid \mathcal{F}\right)$ denote the probability that unit $j$ in matched set $i$ will receive treatment. The Rosenbaum bounds sensitivity analysis considers that two units $i j$ and $i j^{\prime}$ in the same matched set $i$, with the same observed covariates $\mathbf{x}_{i j}=\mathbf{x}_{i j^{\prime}}=\mathbf{x}_{i}$, may differ in their chances of receiving the treatment by at most a factor of $\Gamma \geq 1$ :

$$
\Gamma^{-1} \leq \frac{\pi_{i j}\left(1-\pi_{i j^{\prime}}\right)}{\pi_{i j^{\prime}}\left(1-\pi_{i j}\right)} \leq \Gamma, \quad \text { for all } i \in\{1, \ldots, I\} \text { and } j, j^{\prime} \in\left\{1, \ldots, n_{i}\right\}
$$

Constraint (2.1) is also known as the Rosenbaum bounds (DiPrete and Gangl, 2004). It is clear that the more $\Gamma$ departs from 1 , the more the treatment assignment potentially departs from random assignment. In the Rosenbaum bounds sensitivity analysis, people are interested in the "worstcase" (i.e., the largest possible) p-value reported by a test statistic $T$ given its observed value $t$ under constraint (2.1) (Rosenbaum, 2002). In prac- 
Sharpening the Rosenbaum Bounds

tice, researchers gradually increase the sensitivity parameter $\Gamma$, report the "worst-case" p-value under each $\Gamma$, and find the largest $\Gamma$ such that the "worst-case" p-value exceeds the prespecified level $\alpha$. Such a changepoint $\Gamma$ is called "sensitivity value" and informs the magnitude of potential hidden bias required to alter the causal conclusion (Zhao, 2019).

For example, in a paired study where $n_{i}=2$ for all $i$, a commonly used family of test statistics are sign-score statistics, including McNemar's test and Wilcoxon's signed rank test. Their general form is $T_{\mathrm{ss}}=\sum_{i=1}^{I} d_{i} \sum_{j=1}^{2} c_{i j} Z_{i j}$, where both $d_{i} \geq 0$ and $c_{i j} \in\{0,1\}$ are functions of $\mathbf{R}$ and so are fixed under $H_{0}$. When each $R_{i j}$ is binary, setting $d_{i}=1$ and $c_{i j}=R_{i j}$ gives McNemar's test. For $i=1, \ldots, I$, define $\bar{T}_{\Gamma, i}$ to be independent random variables taking the value 1 with probability $p_{i}^{+}$and the value 0 with probability $1-p_{i}^{+}$, where

$$
p_{i}^{+}= \begin{cases}0 & \text { if } c_{i 1}=c_{i 2}=0 \\ 1 & \text { if } c_{i 1}=c_{i 2}=1 \\ \frac{\Gamma}{1+\Gamma} & \text { if } c_{i 1} \neq c_{i 2} .\end{cases}
$$

As shown in Section 4.3 in Rosenbaum (2002), under the Rosenbaum bounds 2.1), for all $t$ and $\Gamma \geq 1$, we have $\operatorname{pr}\left(T_{\mathrm{ss}} \geq t \mid \mathcal{F}, \mathcal{Z}\right) \leq \operatorname{pr}\left(\sum_{i=1}^{I} \bar{T}_{\Gamma, i} \geq t \mid\right.$ $\mathcal{F}, \mathcal{Z})$. That is, in the Rosenbaum bounds sensitivity analysis, the "worstcase" p-value under $\Gamma$ reported by $T_{\mathrm{ss}}$ given $t$ is $\operatorname{pr}\left(\sum_{i=1}^{I} \bar{T}_{\Gamma, i} \geq t \mid \mathcal{F}, \mathcal{Z}\right)$. 
Sharpening the Rosenbaum Bounds

Assuming no interactions between observed and unobserved covariates, the upper bound $\operatorname{pr}\left(\sum_{i=1}^{I} \bar{T}_{\Gamma, i} \geq t \mid \mathcal{F}, \mathcal{Z}\right)$ is sharp in the sense that it can be achieved for some arrangements of unobserved covariates; see Section 4.3 in Rosenbaum (2002). However, as we will show in Theorem 1 in Section 3 , in the presence of interactions between observed and unobserved covariates, the upper bound $\operatorname{pr}\left(\sum_{i=1}^{I} \bar{T}_{\Gamma, i} \geq t \mid \mathcal{F}, \mathcal{Z}\right)$ induced from the Rosenbaum bounds (2.1) is in general loose in the sense that it cannot be achieved for any arrangements of unobserved covariates. The result in Theorem 1 also holds for general matching regimes (including pair matching, matching with multiple controls, and full matching).

\section{The Rosenbaum bounds are loose in the presence of $X-U$ in-} teractions

The Rosenbaum bounds (2.1) is an odds ratio bound imposed on all matched sets that does not explicitly involve the observed covariates $\mathbf{x}_{i j}$ and a hypothesized unobserved covariate $u_{i j}$; it is natural to consider how it can be derived from a model expressed in terms of $\mathbf{x}_{i j}$ and $u_{i j}$ for the treatment assignment probability $\pi_{i j}($ Rosenbaum, 2002). Since the first paper on the Rosenbaum bounds sensitivity analysis (Rosenbaum, 1987), considering a logit form linking $\pi_{i j}$ to $\mathbf{x}_{i j}$ and $u_{i j}$ with no interactions between $\mathbf{x}_{i j}$ and $u_{i j}$ 
Sharpening the Rosenbaum Bounds

has been a routine way of interpreting the Rosenbaum bounds (2.1) and has been applied in numerous studies (Rosenbaum, 2002; DiPrete and Gangl, 2004):

$$
\log \left(\frac{\pi_{i j}}{1-\pi_{i j}}\right)=g\left(\mathbf{x}_{i j}\right)+\gamma u_{i j}, \quad u_{i j} \in[0,1],
$$

where $\gamma \in \mathbb{R}$ is unknown, and $g(\cdot)$ is an arbitrary unknown function of $\mathbf{x}_{i j}$. Note that the constraint $u_{i j} \in[0,1]$ is no more restrictive than assuming a bounded support of $u_{i j}$ and is only imposed to make the scalar $\gamma$ more interpretable (Rosenbaum, 1987, 2002). For example, if the original unobserved covariate $\widetilde{u}_{i j} \in[0, \xi]$ for some $0<\xi<+\infty$, we just need to consider a normalized unobserved covariate $u_{i j}=\xi^{-1} \widetilde{u}_{i j} \in[0,1]$ and consider the logit model of $\pi_{i j}$ in terms of $\mathbf{x}_{i j}$ and $u_{i j}=\xi^{-1} \widetilde{u}_{i j} \in[0,1]$. It is then straightforward to show that the Rosenbaum bounds (2.1) can be implied from (3.2) with $\Gamma=\exp (|\gamma|)($ Rosenbaum, 2002, DiPrete and Gangl, 2004). Note that the Rosenbaum bounds (2.1) are a type of nonparametric odds ratio bounds which can be implied from more general models on $\pi_{i j}$ than model (3.2) with appropriate equation linking $\Gamma$ and the proposed model on $\pi_{i j}$. In the previous literature, model (3.2) is extensively used as a working model for interpreting the Rosenbaum bounds due to its simplicity and clarity. However, as we will show in the rest of this section, only considering model (3.2) and ignoring potential interactions between observed and 
Sharpening the Rosenbaum Bounds

unobserved covariates can be harmful in a sensitivity analysis.

In this section, we instead consider a more general model of $\pi_{i j}$ in terms of $\mathbf{x}_{i j}$ and $u_{i j}$ allowing for any possible additive two-way interactions between each $\mathbf{x}_{i j(k)}$ and $u_{i j}(X-U$ interactions):

$$
\log \left(\frac{\pi_{i j}}{1-\pi_{i j}}\right)=g\left(\mathbf{x}_{i j}\right)+\beta^{T} \mathbf{x}_{i j} \times u_{i j}+\gamma u_{i j}, \quad u_{i j} \in[0,1],
$$

where $\beta^{T} \in \mathbb{R}^{K}$ and $\gamma \in \mathbb{R}$ are unknown, and $g(\cdot)$ is an arbitrary unknown function of $\mathbf{x}_{i j}$. Similar to the arguments under model (3.2), the constraint $u_{i j} \in[0,1]$ is no more restrictive than assuming a bounded support of $u_{i j}$ and is only imposed to make $\beta^{T}$ and $\gamma$ more interpretable. When $\beta^{T}=\mathbf{0}$ (i.e., no $X$-U interactions), model (3.3) reduces to the original model $(3.2)$ that motivated the Rosenbaum bounds sensitivity analysis (Rosenbaum, 1987). Under (3.3), according to the definition of $\Gamma$ in the Rosenbaum bounds (2.1), the following equation linking the prespecified sensitivity parameter $\Gamma$ and the unknown parameters $\left(\beta^{T}, \gamma\right)$ can be obtained:

$$
\begin{aligned}
\Gamma & =\max _{i, j, j^{\prime}} \frac{\pi_{i j}\left(1-\pi_{i j^{\prime}}\right)}{\pi_{i j^{\prime}}\left(1-\pi_{i j}\right)} \text { subject to } \mathbf{x}_{i j}=\mathbf{x}_{i j^{\prime}} \text { and } u_{i j}, u_{i j^{\prime}} \in[0,1] \text { for all } i, j, j^{\prime} \\
& =\max _{i=1, \ldots, I} \exp \left(\left|\beta^{T} \mathbf{x}_{i}+\gamma\right|\right) .
\end{aligned}
$$

See Appendix for a derivation of equation (3.4). Note that when $\beta^{T}=$ 0, equation (3.4) reduces to the commonly used equation $\Gamma=\exp (|\gamma|)$ obtained under model (3.2). A key insight from equation (3.4) is that, in 
Sharpening the Rosenbaum Bounds

the presence of $X-U$ interactions, setting the sensitivity parameter $\Gamma$ not only incorporates our prior belief on the unknown structural parameters $\left(\beta^{T}, \gamma\right)$, but also information about the matched observed covariates $\mathbf{x}_{i}$, $i=1, \ldots, I$. The following result claims that the Rosenbaum bounds 2.1 will almost inevitably be conservative if there are any interactions between observed and unobserved covariates.

Theorem 1. Consider the sensitivity parameter $\Gamma$ defined in the Rosenbaum bounds (2.1). Let $\Gamma>1$, and suppose that there exist two matched sets $i_{1}$ and $i_{2}$ such that $\mathbf{x}_{i_{1}} \neq \pm \mathbf{x}_{i_{2}}$. Then we have under model (3.3), there exist some $\mathbf{x}^{*} \in \mathbb{R}^{K}$ and a subset $E$ of $\mathbb{R}^{K}$ of Lebesgue measure zero, such that for any $\beta^{T} \neq \mathbf{0}$ (i.e., if there exist any interaction terms between $\mathbf{x}_{i j}$ and $u_{i j}$ ) and $\beta^{T} \notin E$, the Rosenbaum bounds (2.1) are loose for any matched set $i$ with $\mathbf{x}_{i} \neq \pm \mathbf{x}^{*}$, in the sense that for any matched set $i$ with $\mathbf{x}_{i} \neq \pm \mathbf{x}^{*}$ there exists some $\Upsilon_{i}<\Gamma$ such that

$$
\Upsilon_{i}^{-1} \leq \frac{\pi_{i j}\left(1-\pi_{i j^{\prime}}\right)}{\pi_{i j^{\prime}}\left(1-\pi_{i j}\right)} \leq \Upsilon_{i}, \quad \text { for all } j, j^{\prime}
$$

Proofs of all theorems and corollaries in this article are in Appendix. We consider a simple example to illustrate the principle of Theorem 1.

Example 1. Suppose that there is only one observed covariate $x_{i j} \in\{0,1\}$, and also an unobserved covariate $u_{i j} \in[0,1]$. Under model (3.3), we have 
Sharpening the Rosenbaum Bounds

$\log \left(\frac{\pi_{i j}}{1-\pi_{i j}}\right)=g\left(x_{i j}\right)+\beta x_{i j} u_{i j}+\gamma u_{i j}$. According to 3.4 , we have $\Gamma=$ $\max \{\exp (|\gamma|), \exp (|\beta+\gamma|)\}$. It is clear that if $\beta \neq 0$ or $-2 \gamma$, we have $\exp (|\gamma|) \neq \exp (|\beta+\gamma|)$. If $\Gamma=\exp (|\gamma|)>\exp (|\beta+\gamma|)$, then the Rosenbaum bounds (2.1) are loose for any matched set $i$ with the common observed covariate $x_{i}=1$. That is, for all matched set $i$ with $x_{i}=1$, we have

$\Gamma^{-1}<\exp (-|\beta+\gamma|) \leq \frac{\pi_{i j}\left(1-\pi_{i j^{\prime}}\right)}{\pi_{i j^{\prime}}\left(1-\pi_{i j}\right)} \leq \exp (|\beta+\gamma|)<\Gamma$, for all $j, j^{\prime}$ and $u_{i j}, u_{i j^{\prime}} \in[0,1]$.

Similarly, if $\Gamma=\exp (|\beta+\gamma|)>\exp (|\gamma|)$, the Rosenbaum bounds (2.1) are loose for any matched set $i$ with $x_{i}=0$. Therefore, when $\beta \neq 0$, unless $\beta \in\{-2 \gamma\}$ (a subset of $\mathbb{R}$ of Lebesgue measure zero), the Rosenbaum bounds are loose for either all matched sets $i$ with $x_{i}=0$ or all matched sets $i$ with $x_{i}=1$.

\section{Sharper odds ratio bounds accounting for $X-U$ interactions}

In this section, we give new odds ratio bounds that are sharper than the Rosenbaum bounds (2.1) when a researcher is concerned about the possible interaction between a particular observed covariate, say, the $k$ th component $x_{(k)}$ of the observed covariates vector $\mathbf{x}$, and the unobserved covariate $u$. We consider a sub-model of (3.3) which allows for possible interaction term 
linking $x_{(k)}$ and $u$ :

$$
\operatorname{logit}\left(\pi_{i j}\right)=\log \left(\frac{\pi_{i j}}{1-\pi_{i j}}\right)=g\left(\mathbf{x}_{i j}\right)+\widetilde{\beta} \widetilde{x}_{i j(k)} u_{i j}+\gamma u_{i j}, \quad u_{i j} \in[0,1],
$$

where $\widetilde{\beta}, \gamma \in \mathbb{R}$ are unknown, and $g(\cdot)$ is an unknown function of $\mathbf{x}_{i j}$. Again, the unobserved covariate $u_{i j} \in[0,1]$ is normalized to make $\widetilde{\beta}$ and $\gamma$ more interpretable. Each $\widetilde{x}_{i j(k)}=\frac{x_{i j(k)}-\min _{i, j} x_{i j(k)}}{\max _{i, j} x_{i j(k)}-\min _{i, j} x_{i j(k)}} \in[0,1]$ is also normalized to make $\widetilde{\beta}$ more interpretable. Again, note that when $\widetilde{\beta}=0$, model (4.5) reduces to the original model assuming no interaction terms that motivated the Rosenbaum bounds sensitivity analysis Rosenbaum, 1987). In addition to the sensitivity parameter $\Gamma$ defined in 2.1 which quantifies the magnitude of the largest possible bias over all matched sets, when $\Gamma>1$, we introduce another prespecified sensitivity parameter $\lambda$ under model 4.5) as

$$
\lambda=\frac{\frac{\partial \operatorname{logit}\left(\pi_{i j}\right)}{\partial u_{i j}} \mid x_{i j(k)}=\max _{i, j} x_{i j(k)}}{\frac{\partial \operatorname{logit}\left(\pi_{i j}\right)}{\partial u_{i j}} \mid x_{i j(k)}=\min _{i, j} x_{i j(k)}}=\frac{\widetilde{\beta}+\gamma}{\gamma}, \quad \gamma \neq 0 .
$$

The sensitivity parameter $\lambda$ quantifies how distinct the effects of $u$ on the treatment assignment probability can be under the largest and smallest possible values of $x_{(k)}$. Note that when $\widetilde{\beta}=0$ (i.e., no interaction between $x_{(k)}$ and $u$ ), we have $\lambda=1$. Let $\widetilde{x}_{i(k)}$ denote the normalized common covariate $x_{i(k)}$ for matched set $i$, therefore $\widetilde{x}_{i(k)}=\widetilde{x}_{i j(k)}=\widetilde{x}_{i j^{\prime}(k)}$ for all $j, j^{\prime}$. Then we have the following sharper odds ratio bounds. 
Sharpening the Rosenbaum Bounds

Theorem 2. Consider the sensitivity parameter $\Gamma$ defined in the Rosenbaum bounds (2.1) with $\Gamma>1$. Under model (4.5) which allows for possible interaction between the observed covariate $x_{(k)}$ and the normalized unobserved covariate $u$, consider the sensitivity parameter $\lambda$ defined in (4.6). Then we have

$$
\Gamma_{\lambda, i}^{-1} \leq \frac{\pi_{i j}\left(1-\pi_{i j^{\prime}}\right)}{\pi_{i j^{\prime}}\left(1-\pi_{i j}\right)} \leq \Gamma_{\lambda, i} \quad \text { for all } i \in\{1, \ldots, I\} \text { and } j, j^{\prime} \in\left\{1, \ldots, n_{i}\right\}
$$

where

$$
\Gamma_{\lambda, i}= \begin{cases}\Gamma^{\left|(\lambda-1) \tilde{x}_{i(k)}+1\right|} & \text { if }|\lambda| \leq 1, \\ \Gamma^{\left|\left(1-\lambda^{-1}\right) \tilde{x}_{i(k)}+\lambda^{-1}\right|} & \text { if }|\lambda|>1 .\end{cases}
$$

We have $1 \leq \Gamma_{\lambda, i} \leq \Gamma$ for all $i$, and the equality $\Gamma_{\lambda, i}=\Gamma$ holds for matched set $i$ if and only if at least one of the following three conditions holds: (a) $\lambda=1$; (b) $|\lambda| \leq 1$ and $x_{i(k)}=\min _{i} x_{i(k)} ;$ (c) $|\lambda| \geq 1$ and $x_{i(k)}=$ $\max _{i} x_{i(k)}$. The bounds (4.7) are sharp in the sense that for all $i, j, j^{\prime}$, there exist $u_{i j}, u_{i j^{\prime}} \in[0,1]$ such that $\left\{\pi_{i j}\left(1-\pi_{i j^{\prime}}\right)\right\} /\left\{\pi_{i j^{\prime}}\left(1-\pi_{i j}\right)\right\}=\Gamma_{\lambda, i}$.

A key feature of the sharper odds ratio bounds in Theorem2 2 is that they incorporate the information of the observed covariates among the matched samples, which is ignored by the Rosenbaum bounds (2.1). In Table 1 , we present some numerical illustrations of the gap between the sensitivity parameter $\Gamma$ in the Rosenbaum bounds 2.1 and the $\Gamma_{\lambda, i}$ in the sharper 
Sharpening the Rosenbaum Bounds

odds ratio bounds proposed in Theorem 2

Table 1: The $\Gamma_{\lambda, i}$ in the sharper odds ratio bounds proposed in Theorem 2 under various $\Gamma, \lambda$, and $\widetilde{x}_{i(k)} \in[0,1]$. Note that $\Gamma_{\lambda, i}=\Gamma$ when $\lambda=1$.

$\begin{array}{cccccccc}\Gamma=2 & \lambda=\frac{1}{8} & \lambda=\frac{1}{4} & \lambda=\frac{1}{2} & \lambda=1 & \lambda=2 & \lambda=4 & \lambda=8 \\ \widetilde{x}_{i(k)}=0 & 2.00 & 2.00 & 2.00 & 2.00 & 1.41 & 1.19 & 1.09 \\ \widetilde{x}_{i(k)}=\frac{1}{5} & 1.77 & 1.80 & 1.87 & 2.00 & 1.52 & 1.32 & 1.23 \\ \widetilde{x}_{i(k)}=\frac{2}{5} & 1.57 & 1.62 & 1.74 & 2.00 & 1.62 & 1.46 & 1.39 \\ \widetilde{x}_{i(k)}=\frac{3}{5} & 1.39 & 1.46 & 1.62 & 2.00 & 1.74 & 1.62 & 1.57 \\ \widetilde{x}_{i(k)}=\frac{4}{5} & 1.23 & 1.32 & 1.52 & 2.00 & 1.87 & 1.80 & 1.77 \\ \widetilde{x}_{i(k)}=1 & 1.09 & 1.19 & 1.41 & 2.00 & 2.00 & 2.00 & 2.00 \\ \Gamma=3 & \lambda=\frac{1}{8} & \lambda=\frac{1}{4} & \lambda=\frac{1}{2} & \lambda=1 & \lambda=2 & \lambda=4 & \lambda=8 \\ \widetilde{x}_{i(k)}=0 & 3.00 & 3.00 & 3.00 & 3.00 & 1.73 & 1.32 & 1.15 \\ \widetilde{x}_{i(k)}=\frac{1}{5} & 2.48 & 2.54 & 2.69 & 3.00 & 1.93 & 1.55 & 1.39 \\ \widetilde{x}_{i(k)}=\frac{2}{5} & 2.04 & 2.16 & 2.41 & 3.00 & 2.16 & 1.83 & 1.69 \\ \widetilde{x}_{i(k)}=\frac{3}{5} & 1.69 & 1.83 & 2.16 & 3.00 & 2.41 & 2.16 & 2.04 \\ \widetilde{x}_{i(k)}=\frac{4}{5} & 1.39 & 1.55 & 1.93 & 3.00 & 2.69 & 2.54 & 2.48 \\ \widetilde{x}_{i(k)}=1 & 1.15 & 1.32 & 1.73 & 3.00 & 3.00 & 3.00 & 3.00\end{array}$

If the observed covariate $x_{(k)} \in\{0,1\}$ is a binary (dummy) variable, 
Sharpening the Rosenbaum Bounds

model 4.5 reduces to

$$
\operatorname{logit}\left(\pi_{i j}\right)=g\left(\mathbf{x}_{i j}\right)+\widetilde{\beta} x_{i j(k)} u_{i j}+\gamma u_{i j}, \quad x_{i j(k)} \in\{0,1\}, u_{i j} \in[0,1],
$$

and the sensitivity parameter $\lambda$ as defined in (4.6) reduces to

$$
\lambda=\frac{\frac{\partial \operatorname{logit}\left(\pi_{i j}\right)}{\partial u_{i j}} \mid x_{i j(k)}=1}{\frac{\partial \operatorname{logit}\left(\pi_{i j}\right)}{\partial u_{i j}} \mid x_{i j(k)}=0}=\frac{\widetilde{\beta}+\gamma}{\gamma}, \quad \gamma \neq 0
$$

That is, the sensitivity parameter $\lambda$ is simply the ratio of the effect of $u$ on the logit of the treatment assignment probability (denoted as $\partial \operatorname{logit} / \partial u$ ) conditional on $x_{(k)}=1$ to that conditional on $x_{(k)}=0$. Theorem 2 implies the following sharper odds ratio bounds when $x_{(k)}$ is binary.

Corollary 1. Under the same setting as that in Theorem 2, if the observed covariate $x_{(k)} \in\{0,1\}$ is a binary (dummy) variable, we have:

1. If $|\lambda|=1$, then the Rosenbaum bounds (2.1) are sharp for all matched sets, in the sense that for all $i, j, j^{\prime}$, there exist some $u_{i j}, u_{i j^{\prime}} \in[0,1]$ such that $\left\{\pi_{i j}\left(1-\pi_{i j^{\prime}}\right)\right\} /\left\{\pi_{i j^{\prime}}\left(1-\pi_{i j}\right)\right\}=\Gamma$ or $\Gamma^{-1}$.

2. If $|\lambda|<1$, then the Rosenbaum bounds (2.1) are sharp for all matched sets $i$ with $x_{i(k)}=0$. While for all matched sets $i$ with $x_{i(k)}=1$, the Rosenbaum bounds (2.1) can be improved with: for all $i, j, j^{\prime}$ with $x_{i(k)}=1$, we have $\Gamma^{-|\lambda|} \leq\left\{\pi_{i j}\left(1-\pi_{i j^{\prime}}\right)\right\} /\left\{\pi_{i j^{\prime}}\left(1-\pi_{i j}\right)\right\} \leq \Gamma^{|\lambda|}$. 
Sharpening the Rosenbaum Bounds

3. If $|\lambda|>1$, then the Rosenbaum bounds (2.1) are sharp for all matched sets $i$ with $x_{i(k)}=1$. While for all matched sets $i$ with $x_{i(k)}=0$, the Rosenbaum bounds (2.1) can be improved with: for all $i, j, j^{\prime}$ with $x_{i(k)}=0$, we have $\Gamma^{-1 /|\lambda|} \leq\left\{\pi_{i j}\left(1-\pi_{i j^{\prime}}\right)\right\} /\left\{\pi_{i j^{\prime}}\left(1-\pi_{i j}\right)\right\} \leq \Gamma^{1 /|\lambda|}$.

Corollary 1 implies that in the binary covariate case, the sign of the sensitivity parameter $\lambda$ does not matter in a sensitivity analysis. It also implies that in this case the more $|\lambda|$ departs from 1 , the less the treatment assignments can potentially depart from random assignments within some matched sets. When $|\lambda|=1$, the bounds in Corollary 1 reduce to the Rosenbaum bounds (2.1).

Remark 1. Note that even if some observed covariates are not exactly matched for some matched sets, the sharper odds ratio bounds proposed in Theorem 2 and Corollary 1 can still be used as approximate sensitivity bounds as long as the following three conditions hold: 1$)$ the function $g\left(\mathbf{x}_{i j}\right)$ in model (4.5) is a continuous function; 2) the observed covariates are very close among individuals within each matched set $i$ (i.e., $\mathbf{x}_{i j} \approx \mathbf{x}_{i j^{\prime}}$ for all $\left.\left.i, j, j^{\prime}\right) ; 3\right)$ the observed covariates $x_{(k)}$ which can interact with the unobserved covariate are equal among individuals within each matched set (i.e., $x_{i j(k)}=x_{i j^{\prime}(k)}=x_{i(k)}$ for all $\left.i, j, j^{\prime}\right)$. Then the following calculation, combined with repeating the arguments in the proof of Theorem 2 in Appendix, 
can justify the validity of the approximation:

$$
\begin{aligned}
\frac{\pi_{i j}\left(1-\pi_{i j^{\prime}}\right)}{\pi_{i j^{\prime}}\left(1-\pi_{i j}\right)} & =\frac{\exp \left\{g\left(\mathbf{x}_{i j}\right)+\widetilde{\beta} \widetilde{x}_{i j(k)} u_{i j}+\gamma u_{i j}\right\}}{\exp \left\{g\left(\mathbf{x}_{i j^{\prime}}\right)+\widetilde{\beta} \widetilde{x}_{i j^{\prime}(k)} u_{i j^{\prime}}+\gamma u_{i j^{\prime}}\right\}} \\
& =\frac{\exp \left\{g\left(\mathbf{x}_{i j}\right)\right\}}{\exp \left\{g\left(\mathbf{x}_{i j^{\prime}}\right)\right\}} \frac{\exp \left\{\widetilde{\beta} \widetilde{x}_{i j(k)} u_{i j}+\gamma u_{i j}\right\}}{\exp \left\{\widetilde{\beta} \widetilde{x}_{i j^{\prime}(k)} u_{i j^{\prime}}+\gamma u_{i j^{\prime}}\right\}} \\
& \approx \exp \left\{\left(\widetilde{\beta} \widetilde{x}_{i(k)}+\gamma\right)\left(u_{i j}-u_{i j^{\prime}}\right)\right\} \quad\left(\text { since } \mathbf{x}_{i j} \approx \mathbf{x}_{i j^{\prime}} \text { and } \widetilde{x}_{i j(k)}=\widetilde{x}_{i j^{\prime}(k)}=\widetilde{x}_{i(k)}\right) .
\end{aligned}
$$

\section{Implementation of the proposed sharper odds ratio bounds}

As we have shown in Theorem 2 in Section 4 , taking potential $X-U$ interactions can lead to sharper odds ratio bounds than the Rosenbaum bounds (2.1). By implementing the sharper odds ratio bounds proposed in Theorem 2, researchers can perform a less conservative sensitivity analysis than that with directly applying the Rosenbaum bounds. The sharper odds ratio bounds proposed in Theorem 2 can be directly embedded in many of the previous results in the Rosenbaum bounds sensitivity analysis: in many cases, researchers just need to follow the procedure of the Rosenbaum sensitivity analysis except replacing all the sensitivity parameter $\Gamma$ with $\Gamma_{\lambda, i}$ proposed in Theorem 2 in each matched set. For example, the following result shows how applying Theorem 2 to perform a sensitivity analysis with a sign-score statistic $T_{\mathrm{ss}}$ can result in a less conservative "worst-case" pvalue than the one reported by directly applying the Rosenbaum bounds 
sensitivity analysis with $T_{\mathrm{ss}}$.

Corollary 2. Let $T_{s s}=\sum_{i=1}^{I} d_{i} \sum_{j=1}^{2} c_{i j} Z_{i j}$ be a sign-score statistic as in-

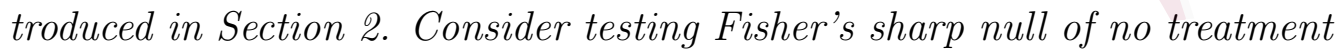
effect $H_{0}$, and the sensitivity parameters $\Gamma$ defined in the Rosenbaum bounds (2.1) and $\lambda$ defined in (4.6) under model (4.5). Define $\Gamma_{\lambda, i}$ as in Theorem 2 and $\bar{T}_{\Gamma, i}$ as in Section 2. For $i=1, \ldots, I$, define $\widetilde{T}_{\Gamma, \lambda, i}$ to be independent random variables taking the value 1 with probability $\widetilde{p}_{\lambda, i}$ and the value 0 with probability $1-\widetilde{p}_{\lambda, i}$, where

$$
\widetilde{p}_{\lambda, i}= \begin{cases}0 & \text { if } c_{i 1}=c_{i 2}=0 \\ 1 & \text { if } c_{i 1}=c_{i 2}=1 \\ \frac{\Gamma_{\lambda, i}}{1+\Gamma_{\lambda, i}} & \text { if } c_{i 1} \neq c_{i 2} .\end{cases}
$$

Then for all $t$ and any fixed $\Gamma>1$ and $\lambda \in \mathbb{R}$, we have $\operatorname{pr}\left(T_{s s} \geq t \mid \mathcal{F}, \mathcal{Z}\right) \leq$ $\operatorname{pr}\left(\sum_{i=1}^{I} \widetilde{T}_{\Gamma, \lambda, i} \geq t \mid \mathcal{F}, \mathcal{Z}\right)$ for any $\mathbf{u} \in[0,1]^{N}$, and we have $\operatorname{pr}\left(\sum_{i=1}^{I} \widetilde{T}_{\Gamma, \lambda, i} \geq\right.$ $t \mid \mathcal{F}, \mathcal{Z}) \leq \operatorname{pr}\left(\sum_{i=1}^{I} \bar{T}_{\Gamma, i} \geq t \mid \mathcal{F}, \mathcal{Z}\right)$. The upper bound $\operatorname{pr}\left(\sum_{i=1}^{I} \widetilde{T}_{\Gamma, \lambda, i} \geq t \mid\right.$ $\mathcal{F}, \mathcal{Z})$ is sharp in the sense that it can be achieved for some $\mathbf{u}$.

\section{Illustration: the effect of anger/hostility tendency on heart problems}

Type A behavior is characterized by hostility, intense ambition, competitive "drive," constant preoccupation with deadlines, and a sense of time 
Sharpening the Rosenbaum Bounds

urgency (Rosenman et al., 1976). Early research data suggested type A behavior was related to heart problems but the original findings have not been supported by subsequent research (Myrtek, 2001). Some researchers have turned their focus to whether tending to be angry and hostile - one of the specific aspects of type A personality - could cause heart problems (Chida and Steptoe, 2009). To study this, we consider data among males from the Wisconsin Longitudinal Study, a long-term study of a random sample of individuals graduated from Wisconsin high schools in 1957 (Herd) et al., 2014). We define a binary indicator of tending to be angry/hostile (i.e., treated) if the respondent said on the 1992-1993 survey (when respondents were approximately 53) that in the last week there were three or more days on which the respondent felt angry or hostile for several hours and 0 (i.e., control) if there were no such day in the last week. We compare high anger/hostility tendency to low anger/hostility tendency and exclude middle levels of anger/hostility tendency because making the treated and control groups sharply differ in dose increases the insensitivity of a study to hidden bias (Rosenbaum, 2004). We take the outcome (heart problem indicator) to be 1 if the respondent reported having had a heart attack, coronary heart disease, or other heart problems in the 2003-2005 survey, and 0 otherwise. We pair match each treated individual with a control on the following 
Sharpening the Rosenbaum Bounds

cardiovascular disease risk factors (Kawachi et al., 1996): age, educational attainment, body mass indicator, drinking alcohol or not, smoking regularly or not, and childhood maltreatment indicator. The childhood maltreatment indicator is 1 if the respondent reported any childhood physical or sexual abuse, and 0 otherwise. Childhood maltreatment has been found to be associated with both anger/hostility tendency and heart problems (Carver et al., 2014; Korkeila et al., 2010), and therefore is a confounder that needs to be controlled for. We discarded all the records with missing outcomes or covariates, and use optimal matching (Rosenbaum, 2010) to match each treated with a control for the six baseline observed covariates, leaving 54 matched pairs. The absolute standardized differences (i.e., difference in means divided by the pooled standard deviation) between the treated and control groups are less than 0.1 for all the six baseline observed covariates, indicating good overall balance (Rosenbaum, 2010). The smoking indicator and childhood maltreatment indicator are exactly matched between the treated unit and control within each matched pair. Although the other four covariates, i.e., age, educational attainment, body mass indicator, and drinking alcohol or not, are not exactly matched between the treated unit and its matched control for all pairs, they are closely matched for most pairs as the correlations for these four covariates equal $0.92,0.98,0.95$, and 0.86 
respectively. Therefore, according to Remark 1, in our study the sharper odds ratio bounds developed in Section 4 can be applied as approximate sensitivity bounds in our sensitivity analysis.

Another covariate we are concerned about as a confounder is the genotype monoamine oxidase A (MAOA) which has been found to be associated with both aggressive behavior and heart disease (McDermott et al., 2009; Kaludercic et al., 2011). The genetic data of the Wisconsin Longitudinal Study is not publicly available, therefore here we treat MAOA genotype as an unobserved covariate. We denote the unobserved MAOA genotype indicator (i.e., the $u$ in model (4.5) to be 1 if the individual has lowactivity MAOA genotype (MAOA-L), and 0 if high-activity MAOA genotype (MAOA-H). According to a controlled experiment done in McDermott et al. (2009), individuals with MAOA-L are more likely to show aggression, suggesting $\gamma>0$ in model (4.5). Childhood maltreatment has been shown to significantly interact with MAOA genotype to confer risk for aggressive behavior: maltreated children with MAOA-L are more likely to develop violent behavior or show hostility (Caspi et al., 2002; Byrd and Manuck, 2014), suggesting that the coefficient of the interaction term $\widetilde{\beta}$ in model 4.5 is greater than 0 and that the sensitivity parameter $\lambda=(\widetilde{\beta}+\gamma) / \gamma>1$. While setting a precise range for $\lambda$ needs further empirical study, some re- 
lated studies suggest that $\lambda \gg 1$. For example, according to Figure $2 \mathrm{~A}$ in Caspi et al. (2002), among severely maltreated (during childhood) males, the logit of probability of conducting disorder among these with MAOA-L is much greater than that among those with MAOA-H. In contrast, among non-maltreated males, these two logits are extremely close. Therefore, if we treat the conducting disorder indicator as a proxy for the anger/hostility tendency indicator, results from Caspi et al. (2002) suggest that $\partial \operatorname{logit} / \partial u$ if maltreated is much greater than that if non-maltreated (i.e., $\widetilde{\beta}+\gamma \gg \gamma$ ), implying $\lambda \gg 1$.

We use Corollary 2 to calculate the "worst-case" p-values $\operatorname{pr}\left(\sum_{i=1}^{I} \widetilde{T}_{\Gamma, \lambda, i} \geq\right.$ $t \mid \mathcal{F}, \mathcal{Z})$ reported by McNemar's test under various $\Gamma$ and $\lambda$, where $\lambda$ quantifies the possible interaction between the childhood maltreatment indicator and MAOA genotype; see Table2. We also report corresponding sensitivity values under various $\lambda$. Note that when $|\lambda|=1$, the "worst-case" p-values are the same as those reported by the Rosenbaum bounds sensitivity analysis. As discussed above, we are particularly concerned about the cases with $\lambda>1$. From Table 2, we can see that through applying the sharper odds ratio bounds developed in Section 4, the "worst-case" p-values are much less conservative than those reported by directly applying the Rosenbaum bounds sensitivity analysis, especially when $\lambda$ is much greater than 1 (i.e., 
Sharpening the Rosenbaum Bounds

Table 2: The "worst-case" p-values reported by McNemar's test under various $\Gamma$ and $\lambda$. When $|\lambda|=1$, they are the same as those reported by the Rosenbaum bounds sensitivity analysis.

$\begin{array}{cccccccc} & |\lambda|=\frac{1}{8} & |\lambda|=\frac{1}{4} & |\lambda|=\frac{1}{2} & |\lambda|=1 & |\lambda|=2 & |\lambda|=4 & |\lambda|=8 \\ \Gamma=1.31 & 0.037 & 0.039 & 0.042 & \mathbf{0 . 0 5 0} & 0.033 & 0.027 & 0.024 \\ \Gamma=1.37 & 0.043 & 0.045 & \mathbf{0 . 0 5 0} & 0.060 & 0.038 & 0.030 & 0.026 \\ \Gamma=1.42 & 0.048 & \mathbf{0 . 0 5 0} & 0.056 & 0.068 & 0.042 & 0.032 & 0.028 \\ \Gamma=1.44 & \mathbf{0 . 0 5 0} & 0.052 & 0.058 & 0.072 & 0.043 & 0.033 & 0.028 \\ \Gamma=1.52 & 0.058 & 0.061 & 0.069 & 0.087 & \mathbf{0 . 0 5 0} & 0.036 & 0.031 \\ \Gamma=1.81 & 0.090 & 0.098 & 0.114 & 0.150 & 0.076 & \mathbf{0 . 0 5 0} & 0.040 \\ \Gamma=2.11 & 0.127 & 0.140 & 0.166 & 0.223 & 0.105 & 0.065 & \mathbf{0 . 0 5 0} \\ \text { Sensitivity value } & 1.44 & 1.42 & 1.37 & 1.31 & 1.52 & 1.81 & 2.11\end{array}$

there is a significant $X-U$ interaction), making a sensitivity analysis significantly more insensitive to hidden bias caused by the potential unobserved covariate. Therefore, for this particular data set, directly applying the Rosenbaum bounds sensitivity analysis can only detect a significant treatment effect up to a moderate magnitude of hidden bias (i.e., $\Gamma=1.31$ ). In contrast, applying our sharper odds ratio bounds to perform a sensitivity analysis allows the researcher to detect a significant treatment effect when 
Sharpening the Rosenbaum Bounds

there is a significant $X-U$ interaction, say, $\lambda \geq 2$, up to a significantly larger magnitude of hidden bias, $\Gamma=1.52$. A bias of $\Gamma=1.5$ is nontrivial as it corresponds to an unobserved covariate that doubles the odds of treatment and increases the odds of a positive treated-minus-control difference in observed outcomes by a factor of 4 (Rosenbaum and Silber, 2009).

\section{Discussion}

We here provide some practical guidance for empirical researchers on when and how our new odds ratio bounds should be used when conducting a Rosenbaum-type sensitivity analysis in matched studies. On the one hand, if a researcher has some prior knowledge about in which direction or to what extent the effects of the concerned unobserved covariate on the treatment assignment probability should vary with different values of the related observed covariate (i.e., a plausible range of the sensitivity parameter $\lambda$ defined in (4.6)), we strongly recommend that, instead of just conducting the sensitivity analysis using the traditional Rosenbaum bounds (2.1) (i.e., setting $\lambda=1$ ), she or he can also report the results of sensitivity analysis under a plausible range of $\lambda$ to better incorporate the expert knowledge to make the sensitivity analysis more informative and less conservative, as shown in Section 6. On the other hand, even if there is no current evidence 
Sharpening the Rosenbaum Bounds

about the existence of any $X-U$ interactions or credible information on the range of $\lambda$ for the concerned $X-U$ interaction term, an empirical researcher could still benefit from our new methods. Suppose a researcher conducted the Rosenbaum bounds sensitivity analysis and found that the "worst-case" p-values $>\alpha=0.05$ even under $\Gamma$ close to 1 , i.e., the sensitivity value is small. Instead of rushing to claim that the causal conclusion is inevitably sensitive to hidden bias, the researcher can diagnose the reasons for sensitivity by selecting some candidate $X-U$ interaction terms and checking the corresponding "worst-case" p-values and sensitivity values under various $\lambda$ through our new odds ratio bound. If the sensitivity values are always small for a reasonably wide range of $\lambda$, then she or he can confirm that the causal conclusion should indeed be sensitive to hidden bias regardless of potential $X-U$ interactions. If instead the sensitivity value becomes substantially larger as $\lambda$ departs from 1 , then this implies that the previous finding that the causal conclusion is sensitive to hidden bias could be due to ignoring the possible $X-U$ interactions, in which case the researcher can do more investigation on the possibility of the actual existence of such $X-U$ interactions to report the sensitivity analysis in a more comprehensive way.

There are limitations to the new odds ratio bound introduced in this work. First, it is only applicable for two-way $X-U$ interactions. For ex- 
ample, if there is an additional three-way interaction term $\widetilde{x}_{i j(k)} \widetilde{x}_{i j\left(k^{\prime}\right)} u_{i j}$ in the treatment assignment probability model (4.5) for some $k \neq k^{\prime}$, then the sensitivity parameter $\lambda$ defined in (4.6) cannot fully capture how the effects of the unobserved covariate $u_{i j}$ on the treatment assignment probability $\pi_{i j}$ would vary with different values of the two observed covariates $\widetilde{x}_{i j(k)}$ and $\widetilde{x}_{i j\left(k^{\prime}\right)}$. Second, when the observed covariate $\widetilde{x}_{i j(k)}$ in the concerned $X-U$ interaction term is not binary, our new odds ratio bound is not applicable if the interaction term in model (4.5) is instead $f\left(\widetilde{x}_{i j(k)}\right) u_{i j}$ where $f\left(\widetilde{x}_{i j(k)}\right)$ is nonlinear in $\widetilde{x}_{i j(k)}$. Third, our new odds ratio bound cannot directly handle multiple $X-U$ interaction terms, e.g., when there are two interaction terms $\widetilde{x}_{i j(k)} u_{i j}$ and $\widetilde{x}_{i j\left(k^{\prime}\right)} u_{i j}$ in model (4.5). Although in principle this type of problems can be solved by introducing additional sensitivity parameters into the odds ratio bounds, doing so can make a sensitivity analysis complicated and hard to interpret. Fourth, in the presence of $X-U$ interactions, after computing the sensitivity value, it requires further study to clarify how large the sensitivity value needs to be to make a study insensitive to hidden bias. Assuming no $X$-U interactions, Hsu and Small (2013) proposed a strategy that calibrates the values of the sensitivity parameters in matched observational studies to the observed covariates to help empirical researchers set plausible ranges of values for the sensitivity parameters. 
Sharpening the Rosenbaum Bounds

It would be helpful to investigate how to extend Hsu and Small (2013)'s approach to the settings allowing $X-U$ interactions. Fifth, in this work, we only consider testing Fisher's sharp null hypothesis and have not discussed testing other types of null hypotheses such as Neyman's weak null hypothesis. In pair-matched observational studies, a promising direction for extending the proposed method in this article to allow testing Neyman's weak null hypothesis is to investigate if the proposed sharper odds ratio bounds proposed in Theorem 2 can be incorporated into the studentized sensitivity analysis framework for the sample average treatment effect developed in Fogarty (2019). Despite these limitations, this work shows how investigating the confounding mechanism more carefully in a matched observational study can make a sensitivity analysis more informative and comprehensive. It might be fruitful for future research to explore how other structural constraints besides the one we explored might be used.

\section{Acknowledgements}

From the second author, Dylan Small:

"I would like to thank Professor Lai for his kind mentoring and friendship throughout my career. When I was a graduate student and interested in applications of statistics to public policy, which was not a focus of any of 
Sharpening the Rosenbaum Bounds

the faculty, Professor Lai took me on and provided me guidance and encouragement to develop my interests. Although I miss the days of being able to find Professor Lai in the math library at all hours, he still always makes the time to talk to me by phone whenever I need advice or encouragement."

The authors would like to thank the participants in the causal inference reading group of the University of Pennsylvania for helpful comments.

\section{Appendix}

\section{A derivation of equation $(3.4)$}

According to the definition of $\Gamma$ in the Rosenbaum bounds (2.1),

$\Gamma=\max _{i, j, j^{\prime}} \frac{\pi_{i j}\left(1-\pi_{i j^{\prime}}\right)}{\pi_{i j^{\prime}}\left(1-\pi_{i j}\right)} \quad$ subject to $\mathbf{x}_{i j}=\mathbf{x}_{i j^{\prime}}$ and $u_{i j}, u_{i j^{\prime}} \in[0,1]$ for all $i, j, j^{\prime}$.

So we have under model (3.3),

$$
\begin{aligned}
\Gamma & =\max _{i, j, j^{\prime}} \max _{u_{i j}, u_{i j^{\prime}} \in[0,1]} \frac{\pi_{i j}\left(1-\pi_{i j^{\prime}}\right)}{\pi_{i j^{\prime}}\left(1-\pi_{i j}\right)} \\
& =\max _{i, j, j^{\prime}} \max _{u_{i j}, u_{i j^{\prime}} \in[0,1]} \frac{\exp \left\{g\left(\mathbf{x}_{i j}\right)+\beta^{T} \mathbf{x}_{i j} \times u_{i j}+\gamma u_{i j}\right\}}{\exp \left\{g\left(\mathbf{x}_{i j^{\prime}}\right)+\beta^{T} \mathbf{x}_{i j^{\prime}} \times u_{i j^{\prime}}+\gamma u_{i j^{\prime}}\right\}} \\
& =\max _{i, j, j^{\prime}} \max _{u_{i j}, u_{i j^{\prime}} \in[0,1]} \exp \left\{\left(\beta^{T} \mathbf{x}_{i}+\gamma\right)\left(u_{i j}-u_{i j^{\prime}}\right)\right\} \quad\left(\text { since } \mathbf{x}_{i j}=\mathbf{x}_{i j^{\prime}}=\mathbf{x}_{i}\right) \\
& =\max _{i, j, j^{\prime}} \exp \left(\left|\beta^{T} \mathbf{x}_{i}+\gamma\right|\right) \\
& =\max _{i=1, \ldots, I} \exp \left(\left|\beta^{T} \mathbf{x}_{i}+\gamma\right|\right) .
\end{aligned}
$$

Therefore the desired equation holds. 
Sharpening the Rosenbaum Bounds

\section{Proof of Theorem 1}

Proof. Let $\mathbf{x}^{*} \in\left\{\mathbf{x}_{1}, \ldots, \mathbf{x}_{I}\right\}$ be an observed covariate vector such that $\Gamma=\max _{i=1, \ldots, I} \exp \left(\left|\beta^{T} \mathbf{x}_{i}+\gamma\right|\right)=\exp \left(\left|\beta^{T} \mathbf{x}^{*}+\gamma\right|\right)>1$. Since there exist two matched sets $i_{1}$ and $i_{2}$ such that $\mathbf{x}_{i_{1}} \neq \pm \mathbf{x}_{i_{2}}$, we have $\left\{\mathbf{x}_{1}, \ldots, \mathbf{x}_{I}\right\} \backslash$ $\left\{\mathbf{x}^{*},-\mathbf{x}^{*}\right\} \neq \emptyset$. For any matched set $i$ such that $\mathbf{x}_{i} \neq \pm \mathbf{x}^{*}$, define the set

$$
E_{i}=\left\{\beta^{T} \in \mathbb{R}^{K}: \exp \left(\left|\beta^{T} \mathbf{x}_{i}+\gamma\right|\right)=\exp \left(\left|\beta^{T} \mathbf{x}^{*}+\gamma\right|\right) \text { and } \beta^{T} \neq \mathbf{0}\right\} .
$$

Since $\mathbf{x}_{i} \neq \pm \mathbf{x}^{*}$, for any $\gamma \in \mathbb{R}$ we have

$E_{i}=\left\{\beta^{T} \in \mathbb{R}^{K}: \beta^{T}\left(\mathbf{x}_{i}-\mathbf{x}^{*}\right)=0\right.$ and $\left.\beta^{T} \neq \mathbf{0}\right\} \cup\left\{\beta^{T} \in \mathbb{R}^{K}: \beta^{T}\left(\mathbf{x}_{i}+\mathbf{x}^{*}\right)+2 \gamma=0\right.$ and $\left.\beta^{T} \neq \mathbf{0}\right\}$

is a subset of $\mathbb{R}^{K}$ of Lebesgue measure zero. Let $E=\underset{i: \mathbf{x}_{i} \neq \pm \mathbf{x}^{*}}{\cup} E_{i}$, then $E$ is

also a subset of $\mathbb{R}^{K}$ of Lebesgue measure zero. For any matched set $i$ such

that $\mathbf{x}_{i} \neq \pm \mathbf{x}^{*}$, when $\beta^{T} \neq \mathbf{0}$ and $\beta^{T} \notin E$, note that

$$
\begin{aligned}
\frac{\pi_{i j}\left(1-\pi_{i j^{\prime}}\right)}{\pi_{i j^{\prime}}\left(1-\pi_{i j}\right)} & =\frac{\exp \left\{g\left(\mathbf{x}_{i j}\right)+\beta^{T} \mathbf{x}_{i j} \times u_{i j}+\gamma u_{i j}\right\}}{\exp \left\{g\left(\mathbf{x}_{i j^{\prime}}\right)+\beta^{T} \mathbf{x}_{i j^{\prime}} \times u_{i j^{\prime}}+\gamma u_{i j^{\prime}}\right\}} \\
& =\exp \left\{\left(\beta^{T} \mathbf{x}_{i}+\gamma\right)\left(u_{i j}-u_{i j^{\prime}}\right)\right\} \quad\left(\text { since } \mathbf{x}_{i j}=\mathbf{x}_{i j^{\prime}}=\mathbf{x}_{i}\right) \\
& \leq \exp \left(\left|\beta^{T} \mathbf{x}_{i}+\gamma\right|\right) \quad\left(\text { since } u_{i j}, u_{i j^{\prime}} \in[0,1]\right) \\
& <\exp \left(\left|\beta^{T} \mathbf{x}^{*}+\gamma\right|\right) \quad(\text { by definition of } E) \\
& =\Gamma,
\end{aligned}
$$

therefore setting $\Upsilon_{i}=\exp \left(\left|\beta^{T} \mathbf{x}_{i}+\gamma\right|\right)$ we have, by symmetry,

$\Gamma^{-1}<\exp \left(-\left|\beta^{T} \mathbf{x}_{i}+\gamma\right|\right) \leq \frac{\pi_{i j}\left(1-\pi_{i j^{\prime}}\right)}{\pi_{i j^{\prime}}\left(1-\pi_{i j}\right)} \leq \exp \left(\left|\beta^{T} \mathbf{x}_{i}+\gamma\right|\right)<\Gamma$, for all $j, j^{\prime}$. 
So the desired result follows.

\section{Proof of Theorem 2}

Proof. Under model 4.5, according to the definition of $\Gamma$ in the Rosenbaum bounds (2.1), we have

$\Gamma=\max _{i, j, j^{\prime}} \frac{\pi_{i j}\left(1-\pi_{i j^{\prime}}\right)}{\pi_{i j^{\prime}}\left(1-\pi_{i j}\right)} \quad$ subject to $\mathbf{x}_{i j}=\mathbf{x}_{i j^{\prime}}$ and $u_{i j}, u_{i j^{\prime}} \in[0,1]$ for all $i, j, j^{\prime}$,

where

$$
\begin{aligned}
\frac{\pi_{i j}\left(1-\pi_{i j^{\prime}}\right)}{\pi_{i j^{\prime}}\left(1-\pi_{i j}\right)} & =\frac{\exp \left\{g\left(\mathbf{x}_{i j}\right)+\widetilde{\beta} \widetilde{x}_{i j(k)} u_{i j}+\gamma u_{i j}\right\}}{\exp \left\{g\left(\mathbf{x}_{i j^{\prime}}\right)+\widetilde{\beta} \widetilde{x}_{i j^{\prime}(k)} u_{i j^{\prime}}+\gamma u_{i j^{\prime}}\right\}} \\
& =\exp \left\{\left(\widetilde{\beta} \widetilde{x}_{i(k)}+\gamma\right)\left(u_{i j}-u_{i j^{\prime}}\right)\right\} \quad\left(\text { since } \mathbf{x}_{i j}=\mathbf{x}_{i j^{\prime}}=\mathbf{x}_{i} \text { and } \widetilde{x}_{i j(k)}=\widetilde{x}_{i j^{\prime}(k)}=\widetilde{x}_{i(k)}\right) \\
& \leq \exp \left(\left|\widetilde{\beta} \widetilde{x}_{i(k)}+\gamma\right|\right) \quad\left(\text { since } u_{i j}, u_{i j^{\prime}} \in[0,1]\right) .
\end{aligned}
$$

Therefore, we have

$$
\begin{aligned}
\Gamma & =\max _{i, j, j^{\prime}} \frac{\pi_{i j}\left(1-\pi_{i j^{\prime}}\right)}{\pi_{i j^{\prime}}\left(1-\pi_{i j}\right)} \quad \text { subject to } \mathbf{x}_{i j}=\mathbf{x}_{i j^{\prime}} \text { and } u_{i j}, u_{i j^{\prime}} \in[0,1] \text { for all } i, j, j^{\prime} \\
& =\max _{i=1, \ldots, I} \exp \left(\left|\widetilde{\beta} \widetilde{x}_{i(k)}+\gamma\right|\right) \\
& =\max \{\exp (|\gamma|), \exp (|\widetilde{\beta}+\gamma|)\} \quad\left(\text { since } \widetilde{x}_{i(k)}=\frac{x_{i(k)}-\min _{i} x_{i(k)}}{\max _{i} x_{i(k)}-\min _{i} x_{i(k)}} \in[0,1]\right) .
\end{aligned}
$$

- Case 1: $|\lambda|=\left|\frac{\widetilde{\beta}+\gamma}{\gamma}\right| \leq 1$. In this case we have $\Gamma=\max \{\exp (|\gamma|), \exp (\mid \widetilde{\beta}+$ 
$\gamma \mid)\}=\exp (|\gamma|)$. Therefore, we have

$$
\begin{aligned}
\frac{\pi_{i j}\left(1-\pi_{i j^{\prime}}\right)}{\pi_{i j^{\prime}}\left(1-\pi_{i j}\right)} & \leq \exp \left(\left|\widetilde{\beta} \widetilde{x}_{i(k)}+\gamma\right|\right) \\
& =\exp \left\{|\gamma| \times\left|\left(\frac{\widetilde{\beta}+\gamma}{\gamma}-1\right) \widetilde{x}_{i(k)}+1\right|\right\} \\
& =\Gamma^{\left|(\lambda-1) \widetilde{x}_{i(k)}+1\right|} \quad\left(\text { since } \Gamma=\exp (|\gamma|) \text { and } \lambda=\frac{\widetilde{\beta}+\gamma}{\gamma}\right) .
\end{aligned}
$$

Therefore, by symmetry we have

$$
\Gamma^{-\left|(\lambda-1) \widetilde{x}_{i(k)}+1\right|} \leq \frac{\pi_{i j}\left(1-\pi_{i j^{\prime}}\right)}{\pi_{i j^{\prime}}\left(1-\pi_{i j}\right)} \leq \Gamma^{\left|(\lambda-1) \widetilde{x}_{i(k)}+1\right|}, \text { for all } i, j, j^{\prime}
$$

and the above bounds are sharp in the sense that the upper bound can be achieved when $u_{i j}-u_{i j^{\prime}}=\operatorname{sign}\left(\widetilde{\beta} \widetilde{x}_{i(k)}+\gamma\right)$, and the lower bound can be achieved when $u_{i j}-u_{i j^{\prime}}=-\operatorname{sign}\left(\widetilde{\beta} \widetilde{x}_{i(k)}+\gamma\right)$, where we let $\operatorname{sign}(x)$ equal 1 if $x>0$, equal -1 if $x<0$, and equal 0 if $x=0$. Since $\widetilde{x}_{i(k)} \in[0,1]$ and $|\lambda| \leq 1$, we have $\Gamma^{\left|(\lambda-1) \widetilde{x}_{i(k)}+1\right|} \leq \Gamma$ and the equality holds if and only if at least one of the following three conditions holds: (a) $\lambda=1$; (b) $x_{i(k)}=\min _{i} x_{i(k)}$ (i.e., $\left.\widetilde{x}_{i(k)}=0\right)$; (c) $\lambda=-1$ and $x_{i(k)}=\max _{i} x_{i(k)}$ (i.e., $\left.\widetilde{x}_{i(k)}=1\right)$.

- Case 2: $|\lambda|=\left|\frac{\widetilde{\beta}+\gamma}{\gamma}\right|>1$. In this case we have $\Gamma=\max \{\exp (|\gamma|), \exp (\mid \widetilde{\beta}+$ 
$\gamma \mid)\}=\exp (|\widetilde{\beta}+\gamma|)$. So we have

$$
\begin{aligned}
\frac{\pi_{i j}\left(1-\pi_{i j^{\prime}}\right)}{\pi_{i j^{\prime}}\left(1-\pi_{i j}\right)} & \leq \exp \left(\left|\widetilde{\beta} \widetilde{x}_{i(k)}+\gamma\right|\right) \\
& =\exp \left\{|\widetilde{\beta}+\gamma| \times\left|\left(1-\frac{\gamma}{\widetilde{\beta}+\gamma}\right) \widetilde{x}_{i(k)}+\frac{\gamma}{\widetilde{\beta}+\gamma}\right|\right\} \\
& =\Gamma^{\left|\left(1-\lambda^{-1}\right) \widetilde{x}_{i(k)}+\lambda^{-1}\right|} \quad\left(\text { since } \Gamma=\exp (|\widetilde{\beta}+\gamma|) \text { and } \lambda=\frac{\widetilde{\beta}+\gamma}{\gamma}\right) .
\end{aligned}
$$

Therefore, by symmetry we have

$$
\Gamma^{-\left|\left(1-\lambda^{-1}\right) \widetilde{x}_{i(k)}+\lambda^{-1}\right|} \leq \frac{\pi_{i j}\left(1-\pi_{i j^{\prime}}\right)}{\pi_{i j^{\prime}}\left(1-\pi_{i j}\right)} \leq \Gamma^{\left|\left(1-\lambda^{-1}\right) \widetilde{x}_{i(k)}+\lambda^{-1}\right|}, \text { for all } i, j, j^{\prime}
$$

and the above bounds are sharp, which is similar to the argument in Case 1. Since $\widetilde{x}_{i(k)} \in[0,1]$ and $|\lambda|>1$, we have $\Gamma^{\left|\left(1-\lambda^{-1}\right) \widetilde{x}_{i(k)}+\lambda^{-1}\right|} \leq \Gamma$ and the equality holds if and only if $x_{i(k)}=\max _{i} x_{i(k)}$ (i.e., $\left.\widetilde{x}_{i(k)}=1\right)$.

The desired result follows from combining the arguments in Case 1 and

Case 2.

\section{Proof of Corollary 1}

Proof. Consider the $\Gamma_{\lambda, i}$ defined in Theorem 2 .

- Case 1: $|\lambda|=1$. In this case, we have $\Gamma_{\lambda, i}=\Gamma^{\left|(\lambda-1) \widetilde{x}_{i(k)}+1\right|}=\Gamma$ for $x_{i(k)} \in\{0,1\}$.

- Case 2: $|\lambda|<1$. In this case, if $x_{i(k)}=0$, we have $\Gamma_{\lambda, i}=\Gamma^{\left|(\lambda-1) \widetilde{x}_{i(k)}+1\right|}=$ $\Gamma$. If $x_{i(k)}=1$, we have $\Gamma_{\lambda, i}=\Gamma^{\left|(\lambda-1) \widetilde{x}_{i(k)}+1\right|}=\Gamma^{|\lambda|}$. 
- Case 3: $|\lambda|>1$. In this case, if $x_{i(k)}=1$, we have $\Gamma_{\lambda, i}=\Gamma^{\left|\left(1-\lambda^{-1}\right) \widetilde{x}_{i(k)}+\lambda^{-1}\right|}=$ $\Gamma$. If $x_{i(k)}=0$, we have $\Gamma_{\lambda, i}=\Gamma^{\left|\left(1-\lambda^{-1}\right) \widetilde{x}_{i(k)}+\lambda^{-1}\right|}=\Gamma^{1 /|\lambda|}$.

Then the desired result follows immediately from applying Theorem 2 .

\section{Proof of Corollary 2}

Proof. The proof follows from a direct adjustment of the proof of Proposition 13 in Rosenbaum (2002). For each fixed $\widetilde{\beta}, \gamma, u_{i j}, i=1, \ldots, I$ and $j=1,2$, the test statistic $T_{\mathrm{ss}}$ is the sum of $I$ independent random variables, where the $i$ th variable equals $d_{i}$ with probability

$$
p_{i}=\frac{c_{i 1} \exp \left\{\left(\widetilde{\beta} \widetilde{x}_{i(k)}+\gamma\right)\left(u_{i 1}-u_{i 2}\right)\right\}+c_{i 2}}{1+\exp \left\{\left(\widetilde{\beta} \widetilde{x}_{i(k)}+\gamma\right)\left(u_{i 1}-u_{i 2}\right)\right\}},
$$

and equals 0 with probability $1-p_{i}$. Note that from the proof of Theorem 2, we have $\Gamma_{\lambda, i}=\exp \left\{\left|\widetilde{\beta} \widetilde{x}_{i(k)}+\gamma\right|\right\}$. Following the proof of Proposition 13 in Rosenbaum 2002), the upper bound distribution $\operatorname{pr}\left(\sum_{i=1}^{I} \widetilde{T}_{\Gamma, \lambda, i} \geq t \mid \mathcal{F}, \mathcal{Z}\right)$ is the distribution of $T_{\mathrm{ss}}$ when $u_{i j}=c_{i j}$ if $\widetilde{\beta} \widetilde{x}_{i(k)}+\gamma \geq 0$ and $u_{i j}=1-c_{i j}$ 


\section{Sharpening the Rosenbaum Bounds REFERENCES}

if $\widetilde{\beta} \widetilde{x}_{i(k)}+\gamma<0$, resulting in the desired

$$
\begin{aligned}
& \widetilde{p}_{\lambda, i}=\left\{\begin{array}{lr}
0 & \text { if } c_{i 1}=c_{i 2}=0, \\
1 & \text { if } c_{i 1}=c_{i 2}=1, \\
\frac{\exp \left\{\left|\widetilde{\beta} \widetilde{x}_{i(k)}+\gamma\right|\right\}}{1+\exp \left\{\left|\tilde{\beta} \widetilde{x}_{i(k)}+\gamma\right|\right\}} & \text { if } c_{i 1} \neq c_{i 2} .
\end{array}\right. \\
& = \begin{cases}0 & \text { if } c_{i 1}=c_{i 2}=0, \\
1 & \text { if } c_{i 1}=c_{i 2}=1, \\
\frac{\Gamma_{\lambda, i}}{1+\Gamma_{\lambda, i}} & \text { if } c_{i 1} \neq c_{i 2} .\end{cases}
\end{aligned}
$$

Applying Theorem 2, the inequality $\operatorname{pr}\left(\sum_{i=1}^{I} \widetilde{T}_{\Gamma, \lambda, i} \geq t \mid \mathcal{F}, \mathcal{Z}\right) \leq \operatorname{pr}\left(\sum_{i=1}^{I} \bar{T}_{\Gamma, i} \geq\right.$

$t \mid \mathcal{F}, \mathcal{Z})$ holds for all $t, \Gamma>1$ and $\lambda \in \mathbb{R}$.

\section{References}

Byrd, A. L. and S. B. Manuck (2014). MAOA, childhood maltreatment, and antisocial behavior: meta-analysis of a gene-environment interaction. Biological Psychiatry 75(1), 9-17.

Carver, C. S., S. L. Johnson, M. E. McCullough, D. E. Forster, and J. Joormann (2014). Adulthood personality correlates of childhood adversity. Frontiers in Psychology 5, 1357.

Caspi, A., J. McClay, T. E. Moffitt, J. Mill, J. Martin, I. W. Craig, A. Taylor, and R. Poulton (2002). Role of genotype in the cycle of violence in maltreated children. Science 297(5582), $851-854$.

Chida, Y. and A. Steptoe (2009). The association of anger and hostility with future coronary 


\section{Sharpening the Rosenbaum Bounds REFERENCES}

heart disease: a meta-analytic review of prospective evidence. Journal of the American College of Cardiology 53(11), 936-946.

DiPrete, T. A. and M. Gangl (2004). 7. Assessing bias in the estimation of causal effects: Rosenbaum bounds on matching estimators and instrumental variables estimation with imperfect instruments. Sociological Methodology 34(1), 271-310.

Ertefaie, A., D. S. Small, and P. R. Rosenbaum (2018). Quantitative evaluation of the tradeoff of strengthened instruments and sample size in observational studies. Journal of the American Statistical Association 113(523), 1122-1134.

Fogarty, C. B. (2019). Studentized sensitivity analysis for the sample average treatment effect in paired observational studies. Journal of the American Statistical Association, 1-13.

Fogarty, C. B. and R. B. Hasegawa (2019). Extended sensitivity analysis for heterogeneous unmeasured confounding with an application to sibling studies of returns to education. The Annals of Applied Statistics 13(2), 767-796.

Gastwirth, J. L., A. M. Krieger, and P. R. Rosenbaum (1998). Dual and simultaneous sensitivity analysis for matched pairs. Biometrika 85(4), 907-920.

Hansen, B. B. (2004). Full matching in an observational study of coaching for the sat. Journal of the American Statistical Association 99(467), 609-618.

Heller, R., P. R. Rosenbaum, and D. S. Small (2009). Split samples and design sensitivity in observational studies. Journal of the American Statistical Association 104(487), 10901101. 


\section{Sharpening the Rosenbaum Bounds REFERENCES}

Herd, P., D. Carr, and C. Roan (2014). Cohort profile: Wisconsin longitudinal study (WLS). International Journal of Epidemiology 43(1), 34-41.

Hsu, J. Y. and D. S. Small (2013). Calibrating sensitivity analyses to observed covariates in observational studies. Biometrics 69(4), 803-811.

Hsu, J. Y., J. R. Zubizarreta, D. S. Small, and P. R. Rosenbaum (2015). Strong control of the familywise error rate in observational studies that discover effect modification by exploratory methods. Biometrika 102(4), 767-782.

Ichino, A., F. Mealli, and T. Nannicini (2008). From temporary help jobs to permanent employment: what can we learn from matching estimators and their sensitivity? Journal of Applied Econometrics 23(3), 305-327.

Kaludercic, N., A. Carpi, R. Menabò, F. Di Lisa, and N. Paolocci (2011). Monoamine oxidases (MAO) in the pathogenesis of heart failure and ischemia/reperfusion injury. Biochimica et Biophysica Acta (BBA)-Molecular Cell Research 1813(7), 1323-1332.

Karmakar, B., B. French, and D. S. Small (2019). Integrating the evidence from evidence factors in observational studies. Biometrika 106(2), 353-367.

Kawachi, I., D. Sparrow, A. Spiro III, P. Vokonas, and S. T. Weiss (1996). A prospective study of anger and coronary heart disease: the normative aging study. Circulation 94(9), 2090-2095.

Korkeila, J., J. Vahtera, K. Korkeila, M. Kivimäki, M. Sumanen, K. Koskenvuo, and M. Koskenvuo (2010). Childhood adversities as predictors of incident coronary heart disease and 


\section{Sharpening the Rosenbaum Bounds REFERENCES}

cerebrovascular disease. Heart 96(4), 298-303.

McDermott, R., D. Tingley, J. Cowden, G. Frazzetto, and D. D. Johnson (2009). Monoamine oxidase a gene (MAOA) predicts behavioral aggression following provocation. Proceedings of the National Academy of Sciences 106(7), 2118-2123.

Myrtek, M. (2001). Meta-analyses of prospective studies on coronary heart disease, type a personality, and hostility. International Journal of Cardiology 79(2-3), 245-251.

Nattino, G. and B. Lu (2018). Model assisted sensitivity analyses for hidden bias with binary outcomes. Biometrics 74(4), 1141-1149.

Neyman, J. S. (1923). On the application of probability theory to agricultural experiments. essay on principles. section 9. (translated and edited by D. M. Dabrowska and T. P. Speed). Statistical Science (1990) 5, 465-480.

Normand, S.-L. T., M. B. Landrum, E. Guadagnoli, J. Z. Ayanian, T. J. Ryan, P. D. Cleary, and B. J. McNeil (2001). Validating recommendations for coronary angiography following acute myocardial infarction in the elderly: a matched analysis using propensity scores. Journal of Clinical Epidemiology 54(4), 387-398.

Ottman, R. (1996). Gene-environment interaction: definitions and study designs. Preventive Medicine 25(6), 764 .

Pérusse, L. and C. Bouchard (1999). Genotype-environment interaction in human obesity/discussion. Nutrition Reviews 57(5), S31. 


\section{Sharpening the Rosenbaum Bounds REFERENCES}

Pimentel, S. D., R. R. Kelz, J. H. Silber, and P. R. Rosenbaum (2015). Large, sparse optimal matching with refined covariate balance in an observational study of the health outcomes produced by new surgeons. Journal of the American Statistical Association 110(510), $515-527$

Rosenbaum, P. R. (1987). Sensitivity analysis for certain permutation inferences in matched observational studies. Biometrika 74, 13-26.

Rosenbaum, P. R. (2002). Observational Studies. Springer.

Rosenbaum, P. R. (2004). Design sensitivity in observational studies. Biometrika 91(1), 153164.

Rosenbaum, P. R. (2010). Design of Observational Studies. Springer.

Rosenbaum, P. R. and J. H. Silber (2009). Amplification of sensitivity analysis in matched observational studies. Journal of the American Statistical Association 104(488), 13981405 .

Rosenman, R. H., R. J. Brand, R. I. Sholtz, and M. Friedman (1976). Multivariate prediction of coronary heart disease during 8.5 year follow-up in the western collaborative group study. American Journal of Cardiology 37(6), 903-910.

Rubin, D. B. (1973). Matching to remove bias in observational studies. Biometrics, 159-183.

Rubin, D. B. (1974). Estimating causal effects of treatments in randomized and nonrandomized studies. Journal of Educational Psychology 66(5), 688. 


\section{Sharpening the Rosenbaum Bounds REFERENCES}

Silber, J. H., S. A. Lorch, P. R. Rosenbaum, B. Medoff-Cooper, S. Bakewell-Sachs, A. Millman,

L. Mi, O. Even-Shoshan, and G. J. Escobar (2009). Time to send the preemie home? additional maturity at discharge and subsequent health care costs and outcomes. Health Services Research $44(2 \mathrm{p} 1), 444-463$.

Stuart, E. A. (2010). Matching methods for causal inference: A review and a look forward. Statistical Science 25(1), 1.

Stuart, E. A. and D. B. Hanna (2013). Commentary: Should epidemiologists be more sensitive to design sensitivity? Epidemiology 24(1), 88-89.

Zhang, B., J. Weiss, D. S. Small, and Q. Zhao (2020). Selecting and ranking individualized treatment rules with unmeasured confounding. Journal of the American Statistical Association, $1-14$.

Zhao, Q. (2019). On sensitivity value of pair-matched observational studies. Journal of the American Statistical Association 114(526), 713-722.

Zubizarreta, J., S. Lorch, G. Marshall, I. D’Apremont, and J. Tapia (2016). Effect of prophylactic cpap in very low birth weight infants in south america. Journal of Perinatology 36(8), 629-634.

Zubizarreta, J. R., M. Cerdá, and P. R. Rosenbaum (2013). Effect of the 2010 chilean earthquake on posttraumatic stress reducing sensitivity to unmeasured bias through study design. Epidemiology (Cambridge, Mass.) 24(1), 79.

Zubizarreta, J. R. and L. Keele (2017). Optimal multilevel matching in clustered observational 


\section{Sharpening the Rosenbaum Bounds REFERENCES}

studies: A case study of the effectiveness of private schools under a large-scale voucher system. Journal of the American Statistical Association 112(518), 547-560.

Graduate Group in Applied Mathematics and Computational Science, School of Arts and Sciences, University of Pennsylvania

E-mail: siyuheng@sas.upenn.edu

Department of Statistics, The Wharton School, University of Pennsylvania

E-mail: dsmall@wharton.upenn.edu 